\title{
Potentials of Capacitive Micromachined Ultrasonic Transducers (CMUT) and Film Bulk Acoustic Wave Resonators (FBAR) for Gas Sensing - an Industrial Point of View
}

\author{
R. Pohle ${ }^{1}$ \\ ${ }^{1}$ Siemens AG, Corporate Technology, Otto-Hahn-Ring 6, D-81739 Munich, Germany \\ roland.pohle@siemens.com
}

\begin{abstract}
Various types of acoustic wave sensors have been investigated for gas sensing applications, but only a view of them are in industrial use until now. This works compares the recent development of two MEMS based acoustic transducers for gas sensors: The electrostatic driven Capacitive Micromachined Ultrasonic Transducer (CMUT) and the piezoelectric Film Bulk Acoustic Resonator (FBAR). Main characteristics of current transducers are compared with respect to their applicability for industrial gas sensors. The detection of $\mathrm{CO}_{2}$ is investigated using a polysiloxane based sensing layer.
\end{abstract}

Key words: Gas sensor, Film Bulk Acoustic Wave Resonators, FBAR, Capacitive Micromachined Ultrasonic Transducer, CMUT.

\section{Introduction}

Gas sensors became a mature technology now and will enable innovations in industrial field like building technology, medical, automotive, consumer and industrial technology. Modern gas sensors are designed in a Microsystems (MEMS) approach to leverage the integration of a complex functionality into a small-sized and low-cost device. Since application requirements and ambient are quite heterogeneous, a wide variety of operating principles and characteristics has to be used. For successful industrialization of a gas sensing application, care has to be taken in advance, that the chosen sensing technology has the potential to fulfill all needs of the intended application.

In this work, MEMS based acoustic sensing technologies are compared, which are on their way to industrialization from the transducer point of view. Even if the focus of both technologies has been more on biological and medical applications in the beginning, the high potential for their use in gas sensors has been already demonstrated. The Capacitive Micromachined Ultrasonic Transducer (CMUT) is available as multi-channel, multi-membrane structure and therefore attractive for many promising applications in the sensor field. Recent work shows the ability of CMUT for sensitive and selective gas detection [1] and methods for optimized sensing coatings have been developed [2].

From the application point of view, the Film Bulk Acoustic Resonator (FBAR) technology provides similar functional opportunities like CMUT, even if the principle of operation and the processes needed for manufacturing are quite different. Recently, a fully integrated FBAR sensor matrix containing 64 pixel has been presented [3].

As an exemplary sensing layer heteropolysiloxane based $\mathrm{CO}_{2}$ sensitive polymer has been chosen, which has already been investigated with capacitive, workfunction and mass sensitive readout [5].

\section{Experimental}

The schematic operation principle of CMUT is depicted in fig. 1: The electrode on the MEMS membrane and the respective counter electrode form a capacitor. The membrane is deflected by a DC bias voltage and actuated by an additive $A C$ voltage $U_{A C}$ to generate ultrasonic oszillations with the amplitude w. Efficient electromechanical coupling is achieved as a result of the strong electric field in the gap. The external medium like gas or liquid is influencing the oscillation of the membrane in the form of changes in capacitance and frequency. For gas sensor applications, the frequency alteration of membrane vibration is investigated, caused by 
gas adsorption on the membrane itself or an additional selective adsorbing layer deposited on the membrane. Typical resonance frequencies are in the $40 \mathrm{MHz}$ range, which allows a certain comparability with results obtained with QMB sensors mainly operated in the several $10 \mathrm{MHz}$ range.

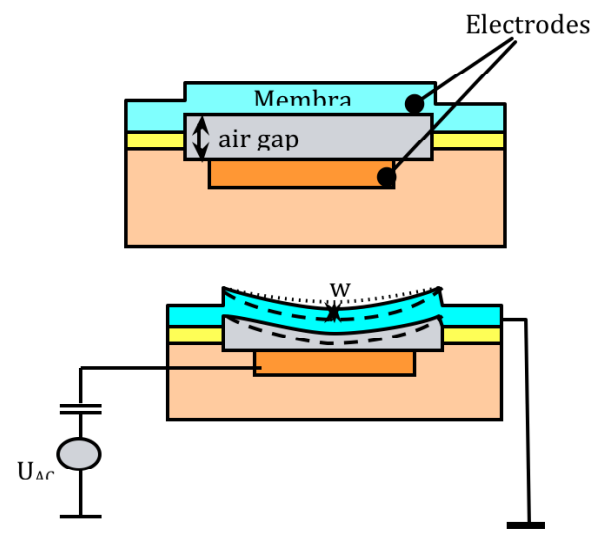

Fig. 1: Schematic view of the CMUT setup.

Fig. 2 (left) shows a CMUT chip with 16 independently operated CMUT arrays. A single CMUT array with polysiloxane sensing coating is displayed in fig. 2(right).
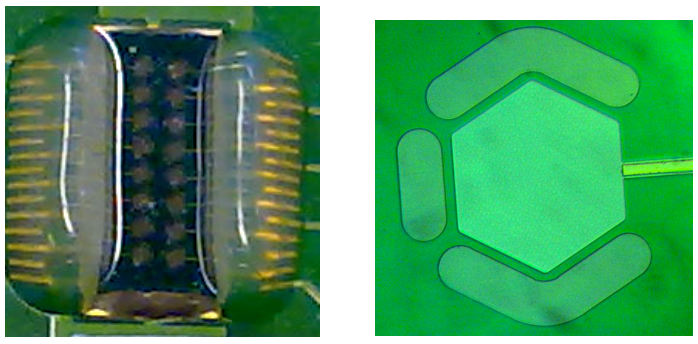

Fig. 2: chip with 16 CMUT arrays (left), single CMUT array with additional gas sensing layer (right).

In comparison to CMUT, the operation frequencies of FBAR are located in the several $100 \mathrm{MHz}$ or $\mathrm{GHz}$ range, so that different acoustic phenomena have to be considered comparing both approaches. The FBAR approach consists of three combined components as depicted in fig. 3: The excitation is realized by piezoelectric actuators, the mechanical energy is transferred with a certain efficiency to an acoustic resonator.

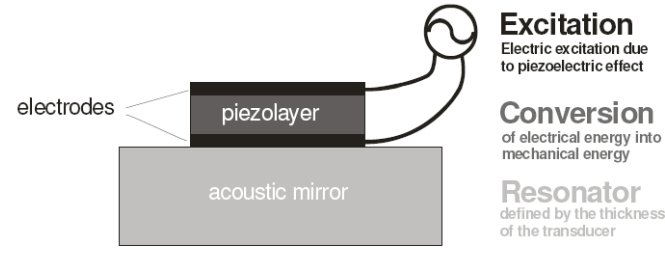

Fig. 3: Schematic view of the CMUT setup [4].

The actual design of the FBAR structure is shown in fig. 4 as described in detail in [3].

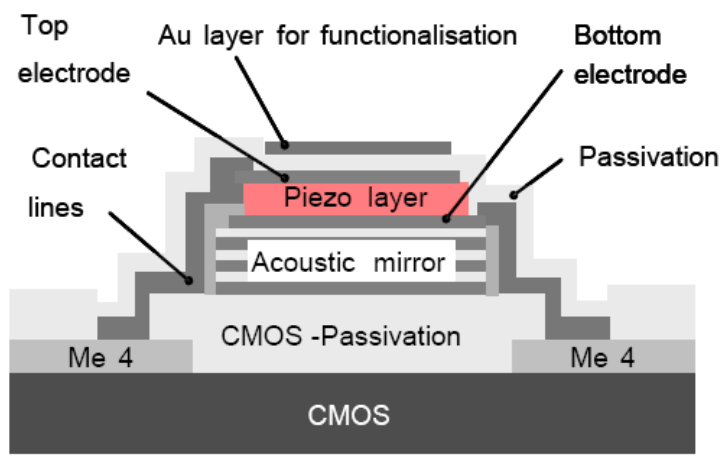

Fig. 4: schematic cross section of the actual FBAR chip [3].

Both, CMUT and FBAR chips have been coated with a hetero-polysiloxane based sensing layer as described in [5]. The resonance frequencies have been evaluated with adapted electronics designed for the particular device.

Measurements were performed at ambient conditions in synthetic air $\left(20 \% \mathrm{O}_{2}, 80 \% \mathrm{~N}_{2}\right)$ at a total flow of $1 \mathrm{l} / \mathrm{min}$ at room temperature (approx. $25^{\circ} \mathrm{C}$ ) with controlled relative humidity. $\mathrm{CO}_{2}$ concentrations in the range from 400ppm to 4000ppm has been applied, according to the concentration range required for room air monitoring.

\section{Results}

To study the influence of the humidity on the gas response of the CMUT device, the response of the resonance frequency to changes in $\mathrm{CO}_{2}$ concentration from 400ppm to 800 pm has been recorded at changing humidity levels (fig. 4). The first effect to remark is the increase in resonance frequency with increasing $\mathrm{CO}_{2}$ concentration. A pure mass effect due to gas adsorption would cause a decrease in resonance frequency with increasing gas concentration. 
In contrast to $\mathrm{CO}_{2}$, an increase in humidity concentration is related to a decreasing resonance frequency. At the actual resonance of the CMUT at approx $40 \mathrm{MHz}$, a change in $\mathrm{CO}_{2}$ by a factor of 2 from $400 \mathrm{ppm}$ to $800 \mathrm{ppm}$ causes a relative frequency shift of approx. $0.1 \%$.

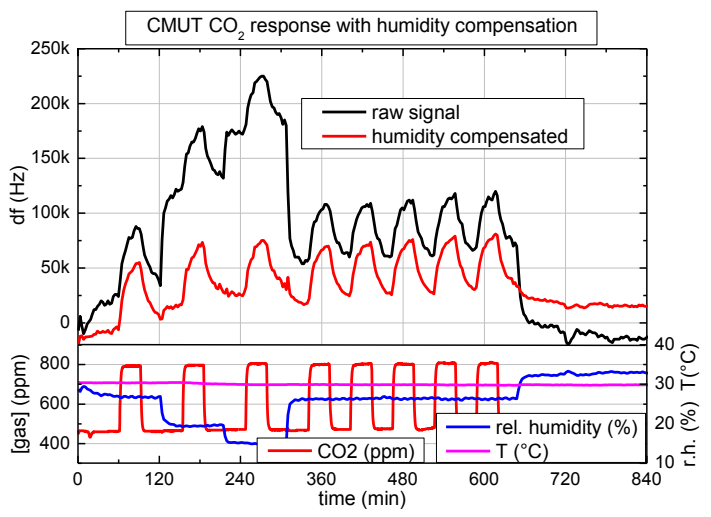

Fig. 4: CO2 response of a CMUT coated with heteropolysiloxane.

Calculating the $\mathrm{CO}_{2}$ concentration from the frequency shift of the CMUT, it is demonstrated, that the CMUT sensor can compete with commercial optical $\mathrm{CO}_{2}$ sensor in regard to resolution of changes in $\mathrm{CO}_{2}$ concentration (fig. 5).

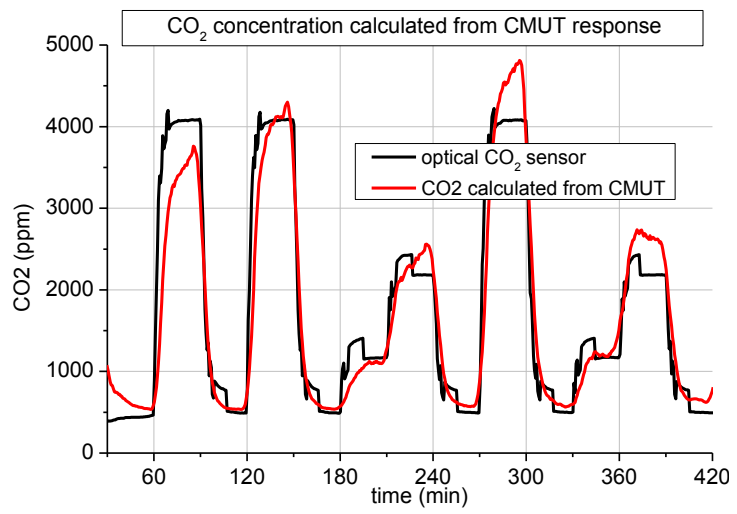

Fig. 5: $\mathrm{CO}_{2}$ response of a CMUT compared to a commercial optical $\mathrm{CO}_{2}$ sensor.

In fig. 6 the frequency shift of an FBAR device exposed to different $\mathrm{CO}_{2}$ concentrations is presented. In contrast to the CMUT sensor, an increase in $\mathrm{CO}_{2}$ level provokes an decrease in frequency as expected from a mass-dominated response. A relative frequency shift of approx. $0.01 \%$ to a change from $400 \mathrm{ppm}$ to $800 \mathrm{ppm}$ $\mathrm{CO}_{2}$ is observed.

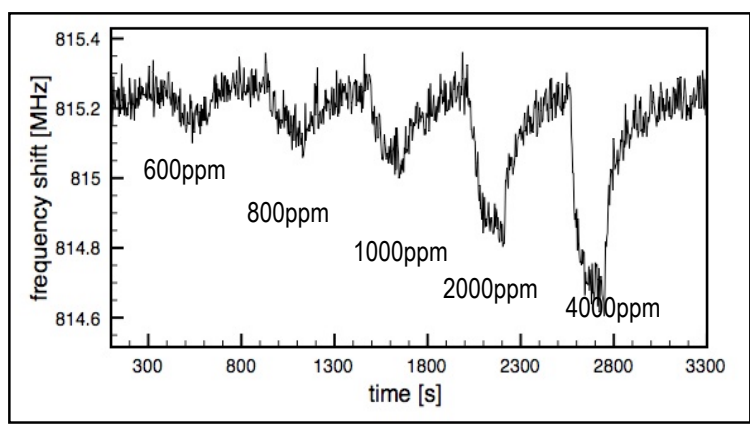

Fig. 6: $\mathrm{CO}_{2}$ response of a FBAR coated with heteropolysiloxane.

\section{Discussion}

Using a polysiloxane sensing layer, both, CMUT and FBAR showed response to changing $\mathrm{CO}_{2}$ levels in room air conditions. Nevertheless, the effects causing the $\mathrm{CO}_{2}$ related shifts in resonance behavior differ to a large extend: The results obtained with the FBAR device correspond to the assumption of a sensing mechanism dominated by mass changes. In contrast, the strong increase of resonance frequency of the CMUT sensors with the $\mathrm{CO}_{2}$ concentration can be related to massive changes in the visco-elastic properties of the polysiloxane layers during changes in the $\mathrm{CO}_{2}$ concentration. In order to quantitatively compare the performance of both approaches,

\section{Summary and Outlook}

Combining MEMS-based CMUT and FBAR transducers with a polysilxane based sensing layer, the detection of changes in $\mathrm{CO}_{2}$ concentration in the range required for room air monitoring has been demonstrated. Based on these results, both technologies are potential candidates for MEMS-based acoustic sensors for room air monitoring.

The FBAR device exhibits a response behavior corresponding to a dominating mass-sensitive effect, while the response of the investigated CMUT sensors is mainly related to gas related changes in the visco-elastic properties of the sensing layer. It has still to be clarified, if the oppositional response is only caused by the different frequencies applied to the sensing layer by the devices, or if the system aspects combining transducer and sensing material have also to be taken into account. In order to quantitatively compare the performance of both approaches in a next step, the influence and coaction of sensing layer, transducer and readout electronics has to be evaluated. 


\section{References}

[1] Lee, H. J.; Park, K. K.; Kupnik,M.; Oralkan, O.; Khuri-Yakub, B. T., Anal. Chem. 2011, 83 (24), 9314-9320.

[2] Y. Li, R. Lucklum, A. Brose, M. Fleischer, Sensors and Actuators B: Chemical 161, 171-177 (2012) doi: 10.1016/j.snb.2011.10.004

[3] K Tukkiniemi, A Rantala, M Nirschl, D Pitzer, T Huber, M Schreiter, Eurosensors 2009, Lausanne doi:10.1016/j.proche.2009.07.262

[4] J. Weber et al., Sensors and Actuators A: Physical 128, 84-88 (2006) doi:10.1016/j.sna.2006.01.005

[5] S. Stegmeier, M. Fleischer, A. Tawil, P. Hauptmann, H.-E. Endres, Sensors and Actuators B: Chemical 154 , 206-212 (2011) doi: 10.1016/j.snb.2009.12.071 